Brit. J. vener. Dis. (1955), 31, 155.

\title{
THE MALE GONORRHOEA “CARRIER"*
}

\section{REPORT OF SEVEN CASES}

BY

\author{
J. B. BITTINER AND G. O. HORNE \\ From the Department of Venereal Diseases, The General Infirmary, Leeds
}

\section{Introduction}

Relatively little attention appears to have been paid to the male gonorrhoea "carrier" (a man with the disease, and capable of transmitting it, but with no symptoms, or with symptoms so slight as to be ignored), although such cases were well known to develop after inadequate sulphonamide treatment. There is little evidence that treatment with penicillin, even in small doses, ever produces a carrier state. However, since gonorrhoea is apparently becoming a much more innocuous disease in both sexes, $\uparrow$ relatively symptomless and asymptomatic male cases may become of importance in its perpetuation.

Attention was drawn to this problem by a patient who first attended the Department of Venereal Diseases, General Infirmary, Leeds, in March, 1953 (Case 1). He was subjected to complete examination in order to establish without doubt that he was a gonorrhoea carrier. Bacteriological investigations were repeated on several occasions before treatment was given in order to confirm the diagnosis, and to allow for the possible development of symptoms. Study of the Department records from the beginning of 1950 revealed five other similar cases, all of whom appeared to be carriers. Although the evidence was less complete in some of them than in Case 1, additional confirmatory investigations (such as examination of the prostatic fluid) had sometimes been done even after a bacteriological diagnosis had been made from the urethra or urine. A seventh case presented in December, 1953, and was fully investigated (Case 7). No further cases have since been encountered.

\footnotetext{
* Received for publication February 4, 1955.

+ Of the last thirty female cases of gonorrhoea seen in the Department of Venereal Diseases, General Infirmary, Leeds, only eleven had genito-urinary symptoms. Seven of these had a vaginal discharge alone, but in three of them this was revealed only at examination because the women were suspected of being infected (two of these seven had also Trichomonas vaginalis vaginitis): only two had urinary symptoms (dysuria and frequency), and they also had a vaginal discharge ; two presented because of vulvar irritation and one of these also had a vaginal discharge (she had trichomonads as well). One of the patients with a discharge and urinary symptoms well). One of the patients with a discharge and urinary symptoms
also had abdominal pain, but no definite clinical evidence of salalso had abdominal pain, but no definite clinical evidence of sal-
pingitis was obtained ; one of those with no genito-urinary symptoms had polyarthritis.
}

Clinical Data

The histories of the seven cases have been kept as brief as possible, and only relevant data included. No history of any genito-urinary symptoms whatsoever could be obtained in four of the patients ; the symptoms of the other three are detailed in the case histories (Cases 5, 6, and 7). All seven denied having received treatment before their investigation.

At each visit the meatus was examined after the urethra had been "stripped", but no abnormality was ever found except at one visit in each of Cases 2 and 6 (see case histories). None of them ever showed any clinical evidence of complications of gonorrhoea. All other findings are recorded in Table I, which includes every visit of each patient. A summary of the diagnostic bacteriological findings is included in the case histories.

\section{Techniques}

Urethral Scraping.-This was obtained by gently scraping the urethral mucous membrane, beyond the fossa navicularis, with a sterilized platinum loop. Smears were stained with Gram's stain, and inoculations made on chocolate agar.

Urine.-This was examined by the standard "twcglass" test. In some cases threads in the urine were removed and examined microscopically after staining; in others the urine was centrifuged and the deposit examined microscopically.

Prostatic Secretion.-The prostate was massaged immediately after the collection of urine. The prostatic secretion was examined as a wet film, stained when abnormal, and also inoculated on chocolate agar. The urine passed after prostatic massage (" prostatic urine") was centrifuged and the deposit examined microscopically.

Bacteriological Diagnosis.-Gonorrhoea was diagnosed in smears on the strength of Gram-negative intracellular diplococci morphologically resembling gonococci; in cultures, on the cultural characteristics of subcultures from chocolate agar (the reliability of the technique has been described earlier (Horne, Bittiner, and Buchanan, 1952) and confirmed since); sugar reactions were carried out in Cases 1 and 7.

\section{Case Histories}

Case 1 (aged 31). - The patient was examined because his wife was found to have a strongly positive gonococcal complement-fixation test whilst under investigation at 
another hospital for "lump in the groin". He denied extra-marital intercourse, as also did his wife, who was subsequently found to have gonorrhoea on examination in the Department.

Prostatic smear and culture were negative at the first visit (urethral scraping not done) but a month later, after it had been confirmed that the patient's wife had gonorrhoea, further investigation revealed positive smear and culture of the urethral scraping and 4 days later, positive urethral scraping, smear, and culture, positivestained thread from urine, prostatic culture, and stained prostatic urine deposit. Three days later, there was further bacteriological confirmation of gonorrhoea (including sugar reactions on culture from urethral scraping).

Case 2 (aged 42). - The patient had been living with the same consort for 5 years, and was investigated because their two children, aged 4 and 2 years, were found to have gonococcal vulvo-vaginitis (confirmed subsequently in the Department). The patient denied having intercourse with anyone other than consort, as also did she. The gonococcus could not be isolated from her. At the patient's first visit the meatus was noted to be abnormally moist, but there was no actual urethral discharge. The evidence suggested that he infected the children whilst in the carrier state. Smear and culture of the urethral scraping, and prostatic smear and culture, were all positive. Trichomonas vaginalis was also found in the urine and prostatic secretion.

Case 3 (aged 25). - The patient came to the Department of his own accord for reassurance with two friends, all of whom had had intercourse with the same consort (not traced). The two friends were both free from infection. The patient admitted that he had recently been exposed to additional risks with other women.

Stained urinary thread and prostatic smear were positive.

He defaulted from observation before test of cure, but it was eventually discovered that he had been living with a consort who was found to have gonorrhoea on examination in the Department.

Case 4 (West African, aged 24).-He was investigated because his regular consort had had two recent attacks of gonorrhoea, diagnosed and treated in the Department. The evidence strongly suggested that he infected the consort whilst in the carrier state.

Prostatic smear and culture were positive (urethral scraping not done). He defaulted before test of cure.

Case 5 (aged 41).-He was investigated because his consort was found to have gonorrhoea on examination in the Department. He admitted exposure with her about a month previously, and stated that about 3 or 4 days after intercourse he had seen a slight urethral discharge, which had lasted for 2 days and had not recurred. There were no other genito-urinary symptoms.

Smear and culture of the urethral scraping, and prostatic culture were all positive.

Case 6 (aged 26). - The patient came to the Department of his own accord because he suspected that 4 months previously he had infected his wife and another consort with gonorrhoea. On questioning he admitted that he had noticed a very slight urethral discharge on several mornings (before micturition) during the few months before reporting. He had no other genitourinary symptoms. He stated that his wife had been treated for "V.D." at a clinic elsewhere 4 months previously; and his consort had been diagnosed as having gonorrhoea on examination in the Department about that time. At the patient's third visit there was a slight mucoid urethral discharge. The evidence strongly suggested that he had infected his wife whilst in the carrier state.

Urethral smear and culture, and prostatic culture were all positive.

Case 7 (aged 26).-He was investigated because his consort had been found in the Department to have gonorrhoea on several occasions during the previous 4 months. Because of the ramifications of the case (several people were involved) it was not possible to be certain when he was infected, but it was known that he was first exposed to the risk of infection at least 4 months previously. He stated that between 6 and 7 weeks previously he had noticed a thick yellow urethral discharge, associated with dysuria and frequency of micturition, and (on one occasion) haematuria. All these symptoms had subsided over a period of 3 weeks, and he denied having received treatment of any type. The evidence strongly suggested that he reinfected his consort whilst in the carrier state.

Positive smear and culture (including sugar reactions) of the urethral scraping, and positive stained urinary deposit were found.

\section{Discussion}

Although difficulties are inevitably associated with the interpretation of information supplied by patients with venereal disease, and with the investigations of such patients, there seems little doubt that the seven cases reported here were gonorrhoea "carriers", and that most of them were probably responsible for spreading the disease while they were symptom-free.

All seven were symptom-free at the time of investigation : four denied ever having had symptoms ; two had had symptoms of urethritis, one marked (Case 7) and one slight (Case 5) but in both the symptoms had cleared up spontaneously; and one (Case 6) had had only mild symptoms of urethritis (an occasional intermittent morning discharge), but, so far as can be ascertained, this was not the reason for his reporting. In fact, in none of the series were symptoms the reason for the investigations.

Despite repeated careful clinical examinations and provocation by various manipulations, no clinical evidence of disease was found at any time in five cases, and only minimal evidence on one occasion in each of two cases (Cases 2 and 6). In 
every case the urine was clear, though sometimes containing a few threads or specks. The evidence suggested that five of the cases had remained in the carrier state for at least a month (Cases 1, 4, 5, 6, and 7), one of them for at least 6 weeks (Case 1), and one for at least 4 months (Case 6).

Bacteriological Diagnosis.-For convenience of discussion salient bacteriological evidence has been summarized in Table II. In five of the cases the diagnosis could be made by the discovery of gonococci in stained urethral smears and/or the growth of gonococci from the same material, although in only one was there any actual urethral discharge, this being very slight and mucoid in character. In another case the diagnosis could be made by the discovery of gonococci in a stained pus thread from the urine (no urethral scraping was examined) ; and in another by the growth of gonococci from the prostatic secretion (no urethral scraping was examined, and the stained urinary deposit showed no gonococci). It is possible that in some cases the organism was a Neisseria other than the gonococcus (sugar reactions were done in only two cases), but this is not relevant to the clinical implications of the cases.

\section{TABLE II}

PRINCIPAL BACTERIOLOGICAL DATA (ABSTRACTED FROM TABLE I)

\begin{tabular}{|c|c|c|c|c|c|c|c|}
\hline \multirow{2}{*}{$\begin{array}{c}\text { Case } \\
\text { No.! }\end{array}$} & \multicolumn{2}{|c|}{$\begin{array}{l}\text { Urethral } \\
\text { Scraping }\end{array}$} & \multirow{2}{*}{\begin{tabular}{|l} 
Urine \\
Stained \\
Thread \\
or \\
Centri- \\
fuged \\
Deposit
\end{tabular}} & \multicolumn{3}{|c|}{$\begin{array}{l}\text { Prostatic } \\
\text { Secretion }\end{array}$} & \multirow{2}{*}{$\begin{array}{l}\text { Prostatic } \\
\text { Urine } \\
\text { Stained } \\
\text { Deposit }\end{array}$} \\
\hline & Smear & Culture & & $\begin{array}{l}\text { Pus } \\
\text { Cells }\end{array}$ & Smear & Culture & \\
\hline 1 & Positive & Positive & (thread) & + & Negative & Positive & Positive \\
\hline 2 & Positive & Positive & - & + & Positive & Positive & $\cdots$ \\
\hline 3 & . & . & (thread) & + & Positive & Negative & $\ldots$ \\
\hline 4 & . & . & (deposit) & \pm & Negative & Positive & $\ldots$ \\
\hline 5 & Positive & & $\begin{array}{l}\text { (thread) } \\
\text { Negative }\end{array}$ & \pm & Negative & Positive & \\
\hline 6 & * Positive & Positive & (deposit) & \pm & & Positive & 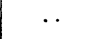 \\
\hline 7 & Negative & Positive & $\begin{array}{l}\text { (deposit) } \\
\text { Positive }\end{array}$ & + & Negative & Negative & Negative \\
\hline
\end{tabular}

* Mucoid urethral discharge.

Positive or Negative for gonococci.

Pus Cells (leucocytes) $+\stackrel{=}{=}$ more than 5 per high-power field. $\pm=$ present but less than 5 per high-power

Significance of Prostatic Cultures.-It is of interest that in five cases gonococci were cultured from the prostatic secretion; in three of these gonococci were also seen in stained films of either the prostatic secretion or of the centrifuged deposit of the prostatic urine (presumably containing prostatic secretion). Care must be taken in interpreting this observation, and it may not be justifiable to conclude that the prostate gland was infected in all these cases. In four of the five cases with a positive prostatic culture it was known that the urethra was also culturally positive. It is possible that, despite the passing of urine, the prostatic material was contaminated by the urethra during its collection.

On the other hand, there is complete concordance in the prostatic fluid between the occurrence of gonococci and the pus cell content. In Cases 1, 2, and 3, where there were gonococci in stained smears, there were also excess pus cells (more than five per high-power field). In spite of the limited significance that can be attached to the pus cell content of the prostatic fluid as examined by the technique used here (Horne, 1955) this is evidence that prostatic infection, as well as urethral infection, was present in these cases. Since neither excess pus cells nor gonococci were seen in stained smears of Cases 4, 5, and 6, the positive prostatic culture may not mean that the prostate was infected.

Gonococcal Complement-Fixation Test (GCFT).In three cases the GCFT was done once only : in two of these (Cases 3 and 4) it was negative, and in the other (Case 6) it was doubtful positive. In another three cases $(2,5$, and 7$)$ the GCFT was strongly positive on at least one occasion. In the last (Case 1) it was doubtful positive on three occasions. This illustrates the limited value of the GCFT in such cases.

Effects of Treatment.-Because of various circumstances different amounts of penicillin were given, ranging from 300,000 units of a procaine preparation to 3.6 million units of a combined procaine and crystalline preparation (Table I, overleaf). There is no reason to believe that a carrier should be more resistant to treatment than a symptomatic case, and there was no evidence in this series of cases that the treatment given was inadequate.

The period of observation after treatment varied (Table I), but five cases had at least one test of cure which included culture of the prostatic secretion. Unfortunately, with the exception of Cases 1 and 7 (who had repeated elaborate tests), examination of the urethral scraping was not done after treatment, since the importance of this was not appreciated at the time. However, no evidence has been obtained that any of the patients have since had a clinical relapse, nor is there evidence that they have since been responsible for infecting others.

Conclusions.-It seems therefore that, in addition to the many patients encountered nowadays with very mild clinical gonorrhoea, there are some men who have a sub-clinical infection in whom the diagnosis can be made only by very careful bacteriological 
TABLE I

SUMMARY OF INVESTIGATIONS

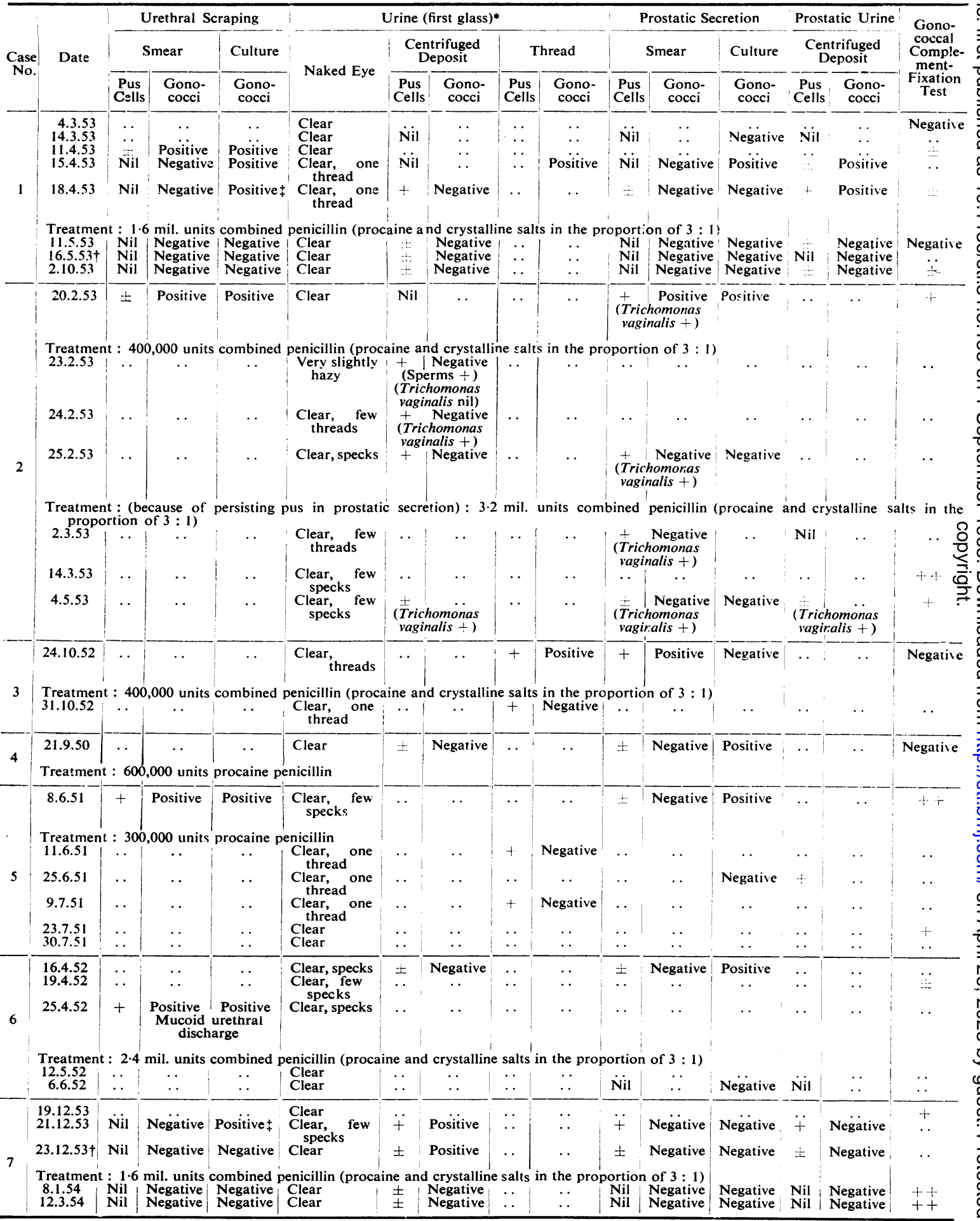


investigation. When such a case is suspected the investigations should include a stained smear and culture of at least one of the following : urethral scraping, urinary threads (if present), centrifuged urine deposit, prostatic secretion, and centrifuged prostatic urine deposit. The likelihood of success will obviously depend on the standard of techniques used, particularly for culturing the gonococcus. In view of the implications usually associated with gonorrhoea, and especially if the patient is symptomfree, more than one positive result should be sought if possible before a diagnosis is made. A GCFT should always be done in such cases, but the limitations of this test have been referred to, and it should not be relied on for a diagnosis.

The importance of the male "carrier" should not be overrated, but in areas where the incidence of gonorrhoea is increasing, or is considered not to be declining rapidly enough as a result of currently used methods of control, the possibility of male "carriers" contributing to the infectious pool (usually attributed only to females) should be considered.

\section{Summary}

1. Attention is drawn to the existence of the male gonorrhoea "carrier" (a man with the disease, and capable of transmitting it, but with no symptoms or with symptoms so slight as to be ignored). Seven such cases were encountered in 4 years in a venereal diseases clinic.

2. These cases are described, and the clinical and bacteriological evidence supporting the diagnosis is presented. The examinations necessary for the investigation of such cases are reviewed.

3. The implications of the male gonorrhoea "carrier" are discussed.

The cultural and serological investigations were carried out in the Bacteriological Laboratory, School of Medicine, Leeds, under the direction of Professor J. W. McLeod, and latterly of Professor C. L. Oakley.

\section{REFERENCES}

Horne, G. O. (1955). Brit. J. Urol., 27, 61.

_-, Bittiner, J. B., and Buchanan, J. R. G. (1952). British Journal of Venereal Diseases, 28, 189.

Footnotes to Table I

Urine

*The second glass in the two-glass test was clear and free from threads and specks on every occasion

"Threads" = coiled threads more than a few mm. long

"Specks" = all other, smaller particles

Pus Cells (leucocytes)

$+=$ more than 5 per high-power field

$\pm=5$ or less per high-power field
Gonococcal Complement-fixation Test

$\pm=$ Doubtful positive

$+=$ Slightly positive

$++=$ Strongly positive

†Anterior urethroscopy-normal

†Sugar reactions-gonococci 